\title{
VERSATILE ANALYSIS OF LIMITED PLASTICITY BY BODY FORCE METHOD
}

Received: date / Accepted: date

\begin{abstract}
A problem of thermally-induced residual stress under plane strain constraint is considered based on a Body Force Method (BFM). As a numerical example, a simple problem of limited plasticity due to a uniform strength of transient line heat source of finite width, which is applied to a surface of a semi-infinite solid for a short duration of time, was considered. Although the out-of-plane component of normal stress $\left(\sigma_{z z}\right)$ can be simply estimated from the in-plane normal stress components $\sigma_{x x}, \sigma_{y y}$ and the Poisson's ratio $\nu$ as $\sigma_{z z}=\nu\left(\sigma_{x x}+\sigma_{y y}\right)$ for elastic plane strain problems, this relation violated when plasticity is considered. As a result, the residual stress in the direction of out-of-plane found to be a major component in the present problem.
\end{abstract}

Keywords Principle of Superposition · Limited Plasticity · Semi-Analytical Approach · Body Force Method · Welding

\section{Introduction}

Most mechanical and structural components are supposed to be used within an extent of linear elasticity, however, due to a presence of indispensable stress concentrators, such as key-ways and notches, an occurrence of limited amount of plasticity have to be tolerated. In the present paper, the word limited plasticity is used for the situation that the relatively small plastic region is surrounded by an elastic foundation so that a plastic flow is restricted

\author{
A. Saimoto \\ Department of Mechanical Systems Engineering, Nagasaki University, 1-14 \\ Bunkyo-machi, Nagasaki 8528521, Japan \\ Tel.: +82(0)95-8192493 \\ Fax: +82(0)95-8192493 \\ E-mail: s-aki@nagasaki-u.ac.jp \\ Y. Imai \\ Department of Mechanical Systems Engineering, Nagasaki University
}


considerably and thereby the magnitude of plastic strain remains in the same order to that of the elastic strain. In order to evaluate the degree of plastic deformation and residual stresses precisely, employment of the commercial codes of finite element (FE) packages that examine full elastic-plastic computation became quite popular in recent years. On the other hand, the use of elastic-plastic FE code is sometimes inefficient because it can not take into account the fact that most part of the component would remain in an elastic state. The other way to evaluate the limited plasticity is often referred as a semi-analytical approach in which the elastic analysis is carried out first and then the result is used to estimate the local plasticity by using the Neuber-Glinka formulas. The latter method is quite successful when the material flow occurs under deformation plasticity, however, this approach sometimes brings a considerable error since the real materials often exhibit the incremental nature of plasticity.

In order to treat problems including limited plasticity effectively, Blomerus and Hills proposed a dislocation based technique[1]. In their method, edge dislocations are distributed into the direction of maximum shear in order to express the occurrence of plastic flow. The magnitude of the Burgers vector at the each material point where the plastic flow occurred is determined through the iterative procedure considering the yield criterion. Since the magnitude of Burgers vector at each material point is determined incrementally, the stress redistribution due to yielding can be modeled sufficiently. On the other hand, the dislocation approach sometimes exhibits a convergence problem in which the direction of maximum shear stress varied frequently due to the fluctuation of the magnitude of Burgers vector. One of the other semi-analytical approach to treat the limited plasticity was proposed by D.H. Chen and H. Nisitani. They employed a force doublet embedded in an elastic continuum in order to express the inelastic strain[2-4]. Although their method is useful for wide range of limited plasticity, it seems difficult to apply to the problem of a special type of the plane strain in which the component of residual stress in the thickness direction becomes the major component. The major difficulty in the analysis of elastic-plastic problem by BFM under plane strain condition arises from the following facts; (i) the increment of plastic strain in the out-of-plane direction estimated from the Prandtl-Reuss flow rule has to be considered even under two-dimensional analysis, and at the same time, (ii) the total strain in the out-of-plane direction has to be zero due to the constraint of plane strain. Moreover, usual elastic-plastic analysis requires one-to-one correspondence between the equivalent stress and the equivalent plastic strain so that the treatment of non-hardening material is considerably difficult.

In the present study, in order to propose a semi-analytical technique for treating the limited plasticity under welding, the method for expressing the plastic strain which occurs not only in the in-plane but also in the out-ofplane direction holding a plain strain constraint is mainly discussed. As an numerical example, limited plastic flow due to over heating by line heater in a non-hardening material, which can be regarded as a simple model of line welding, is considered. 


\section{A brief introduction of BFM}

BFM was originally proposed in 1967 as a boundary type method for elastic stress analysis[5]. In BFM. any elastic problem is expressed in terms of the superposition of fundamental solutions of elasticity. As a fundamental solution, stress field due to an isolated point force acting in an infinite elastic body (usually referred as Kelvin solution) is preferably employed due to its simplicity. In fact, based on the principle of the BFM, stress components at an arbitrary point $P, \sigma_{i j}(P)$, in an elastic medium can be written as,

$$
\sigma_{i j}(P)=\sigma_{i j}^{0}(P)+\int_{\Gamma} \sigma_{i j}\left(P, Q_{k}\right) \phi_{k}(Q) d \Gamma(Q)
$$

where $P \in R$ is an arbitrary point in the reference region $R$ which is surrounded by the imaginary boundary $\Gamma$. $Q \in \Gamma$ is a source point which lies and moves on $\Gamma \cdot \sigma_{i j}\left(P, Q_{k}\right)$ is a fundamental stress solution (stress component $\sigma_{i j}$ at a point $P$ caused by a unit magnitude of point force acting into $k$-direction $(k=x, y)$ at a source point $Q)$ and $\phi_{k}(Q)$ is a density of the $k$-component of body force which has to be determined so that the given boundary conditions are satisfied.

Consider an infinite sheet with a circular hole of diameter $2 a$, subjected to external tensile stresses as illustrated in Fig.1. In this example, the reference region $R$ is an infinite plate excluding a circular disk of radius $a$, therefore, the imaginary boundary $\Gamma$ is a circular ring of diameter $2 a$. The stress component at a point $P$ can then be expressed according to Eq.(1) as,

$$
\begin{aligned}
& \sigma_{x x}(P)=\sigma_{x x}^{0}+\int_{\Gamma}\left\{\sigma_{x x}\left(P, Q_{x}\right) \phi_{x}(Q)+\sigma_{x x}\left(P, Q_{y}\right) \phi_{y}(Q)\right\} d \Gamma(Q) \\
& \sigma_{y y}(P)=\sigma_{y y}^{0}+\int_{\Gamma}\left\{\sigma_{y y}\left(P, Q_{x}\right) \phi_{x}(Q)+\sigma_{y y}\left(P, Q_{y}\right) \phi_{y}(Q)\right\} d \Gamma(Q) \\
& \sigma_{x y}(P)=\int_{\Gamma}\left\{\sigma_{x y}\left(P, Q_{x}\right) \phi_{x}(Q)+\sigma_{x y}\left(P, Q_{y}\right) \phi_{y}(Q)\right\} d \Gamma(Q)
\end{aligned}
$$

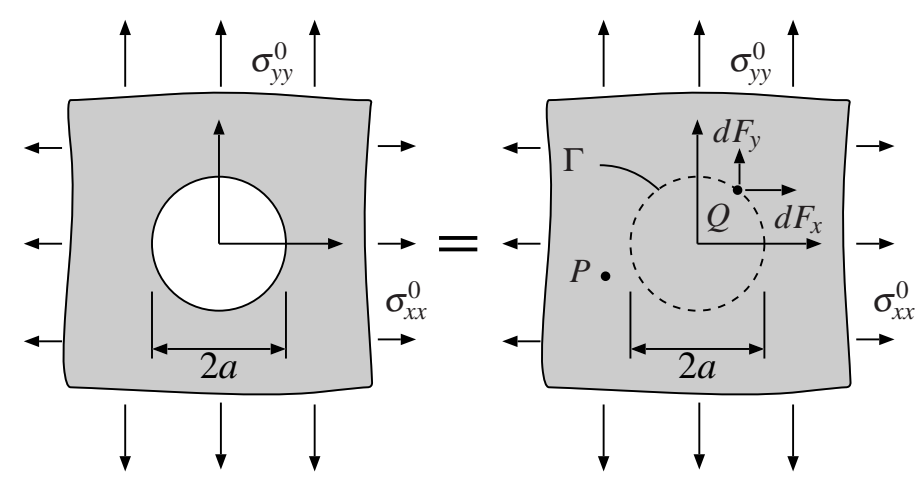

Fig. 1 Analysis of an elastic sheet having a circular hole of diameter $2 a$, subjected to external tensile stresses $\sigma_{x x}^{0}$ and $\sigma_{y y}^{0}$ at infinity 
in which $\sigma_{x x}^{0}$ and $\sigma_{y y}^{0}$ are the uniform tensile stresses at infinity. $\phi_{x}(Q)$ and $\phi_{y}(Q)$ are the unknown densities of body forces which define the magnitude of body forces at point $Q$ per unit length of an imaginary boundary as,

$$
d F_{x}(Q)=\phi_{x}(Q) d \Gamma, d F_{y}(Q)=\phi_{y}(Q) d \Gamma
$$

In numerical analysis, the imaginary boundary $\Gamma$ is divided into several segments and the density of body forces at each segment is determined numerically. If the problem is rather simple, the unknown density of body forces have closed form solution and can be determined theoretically. In fact, the situation illustrated in Fig.1 is one of a such case.

It is well known that two-dimensional elasticity problem can be expressed in terms of two complex potentials $\Omega(z)$ and $\omega(z)$ such that,

$$
\begin{aligned}
\sigma_{x x}+\sigma_{y y} & =2\left\{\Omega^{\prime}(z)+\overline{\Omega^{\prime}(z)}\right\} \\
\sigma_{y y}-\sigma_{x x}+2 i \sigma_{x y} & =2\left\{\bar{z} \Omega^{\prime \prime}(z)+\omega^{\prime}(z)\right\}
\end{aligned}
$$

where $z$ is a complex variable that represents the reference point $z=x+i y$, $i$ is an imaginary unit and the over-bar denotes the complex conjugate. The Kelvin solution (stress field at a point $z$ due to a point force of magnitudes $F_{x}$ and $F_{y}$ acting at a source point $\zeta=\xi+i \eta$ in an infinite elastic sheet) can be expressed in terms of complex potentials as,

$$
\begin{aligned}
& \Omega(z)=-\frac{F_{x}+i F_{y}}{2 \pi(\kappa+1)} \log (z-\zeta) \\
& \omega(z)=\frac{\kappa\left(F_{x}-i F_{y}\right)}{2 \pi(\kappa+1)} \log (z-\zeta)+\frac{F_{x}+i F_{y}}{2 \pi(\kappa+1)} \frac{\bar{\zeta}}{z-\zeta}
\end{aligned}
$$

where $\kappa$ is a constant relating to Poisson's ratio $\nu$ as $\kappa=(3-\nu) /(1+\nu)$ for plane stress and $\kappa=3-4 \nu$ for plane strain. By using the complex potentials, the elastic fields of Fig.1 can be written as,

$$
\begin{aligned}
\Omega(z) & =\frac{\sigma_{x x}^{0}+\sigma_{y y}^{0}}{4} z-\oint_{\Gamma} \log \left(z-a e^{i \theta}\right) \frac{\phi_{x}(\theta)+i \phi_{y}(\theta)}{2 \pi(\kappa+1)} a d \theta \\
\omega(z) & =\frac{\sigma_{y y}^{0}-\sigma_{x x}^{0}}{2} z+\oint_{\Gamma} \log \left(z-a e^{i \theta}\right) \frac{\kappa\left\{\phi_{x}(\theta)-i \phi_{y}(\theta)\right\}}{2 \pi(\kappa+1)} a d \theta \\
& +\oint_{\Gamma} \frac{a e^{-i \theta}}{z-a e^{i \theta}} \frac{\phi_{x}(\theta)+i \phi_{y}(\theta)}{2 \pi(\kappa+1)} a d \theta
\end{aligned}
$$

since the source point $\zeta(=Q)$ lies on the imaginary circle of radius $a$, which can be expressed as $\zeta=a e^{i \theta}$. In the problem shown in Fig.1, the density functions $\phi_{x}(\theta)$ and $\phi_{y}(\theta)$ have closed form solution such that;

$$
\begin{aligned}
& \phi_{x}(\theta)=\frac{\kappa+1}{2(\kappa-1)}\left\{\kappa \sigma_{x x}^{0}-(\kappa-2) \sigma_{y y}^{0}\right\} \cos \theta=\text { constant, } \rho_{x} \times \cos \theta(12) \\
& \phi_{y}(\theta)=\frac{\kappa+1}{2(\kappa-1)}\left\{\kappa \sigma_{y y}^{0}-(\kappa-2) \sigma_{x x}^{0}\right\} \sin \theta=\text { constant, } \rho_{y} \times \sin \theta(13)
\end{aligned}
$$


In fact, substituting Eqs.(12), (13) into Eqs.(10), (11) and then by examining the contour integral considering $|z|>a$ using Cauchy's integral theorem, the exact expressions of complex potentials of Fig.1 are obtained as,

$$
\begin{aligned}
& \Omega(z)=\frac{\sigma_{x x}^{0}+\sigma_{y y}^{0}}{4} z+\frac{\sigma_{x x}^{0}-\sigma_{y y}^{0}}{2} \frac{a^{2}}{z} \\
& \omega(z)=\frac{\sigma_{y y}^{0}-\sigma_{x x}^{0}}{2} z-\frac{\sigma_{x x}^{0}+\sigma_{y y}^{0}}{2} \frac{a^{2}}{z}+\frac{\sigma_{x x}^{0}-\sigma_{y y}^{0}}{2} \frac{a^{4}}{z^{3}}
\end{aligned}
$$

It should be noted that Eqs.(14) and (15) are the strict solution of the classical elasticity problem shown in Fig.1. That is, BFM can be considered not a mere of numerical method for elastic stress analysis but a solution method that often has a possibility to bring even a closed form strict solution of the elastic problems.

It is readily found that the density functions of the body force in Eqs.(12) and (13) are given by the product of some constant and the components of unit outward normal $(\cos \theta, \sin \theta)$ at a point $Q$ on the imaginary boundary $\Gamma$. Therefore, the expression of boundary integral in Eqs.(10) and (11) can be transformed into a form of area integral by using the Green's theorem as,

$$
\begin{aligned}
\Omega(z) & =\frac{\sigma_{x x}^{0}+\sigma_{y y}^{0}}{4} z+\frac{1}{2 \pi(\kappa+1)} \iint_{\bar{R}} \frac{\rho_{x}-\rho_{y}}{z-\zeta} d \xi d \eta \\
\omega(z) & =\frac{\sigma_{y y}^{0}-\sigma_{x x}^{0}}{2} z-\frac{\kappa-1}{2 \pi(\kappa+1)} \iint_{\bar{R}} \frac{\rho_{x}+\rho_{y}}{z-\zeta} d \xi d \eta \\
& +\frac{1}{2 \pi(\kappa+1)} \iint_{\bar{R}}\left(\rho_{x}-\rho_{y}\right) \frac{\bar{\zeta}}{(z-\zeta)^{2}} d \xi d \eta
\end{aligned}
$$

in which $\bar{R}$ is a region inside of the imaginary boundary $\Gamma$, which is referred as an auxiliary region. Equivalence of Eqs.(10), (11) and Eqs.(16), (17) directly implies that the influence of the body force applied along the imaginary boundary is equivalent to that of due to embedded force doublets into the auxiliary region. The physical meaning of the force doublet is an embedded eigen stress at a point where it is applied. In a problem that includes any of inelastic strain such as plastic strain, the force doublet is used to express its influence since the eigen stress directly relates to the eigen strain through the

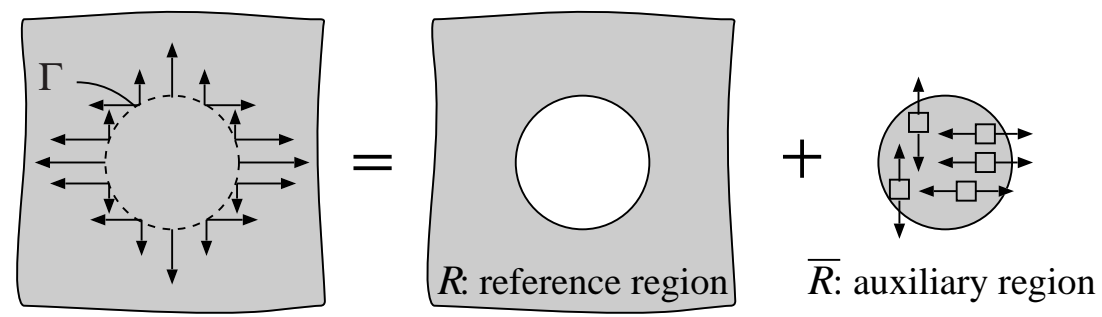

Fig. 2 The reference and auxiliary regions (distribution of body force along an imaginary boundary $\Gamma$ for the problem of Fig.1 can be replaced by the distribution of uniform force doublets embedded inside of an auxiliary region $\bar{R}$ ) 


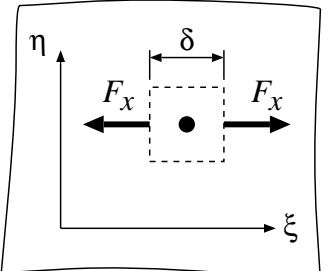

(a) doublet $T_{x x}$

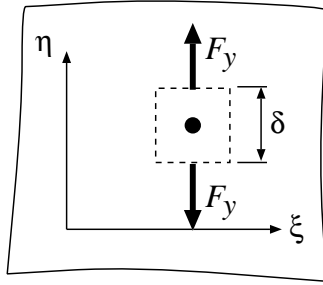

(b) doublet $T_{y y}$

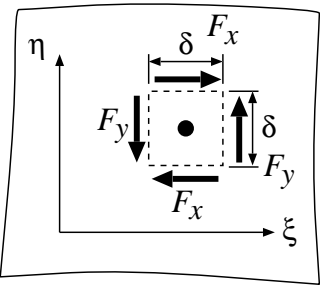

(c) doublet $T_{x y}$

Fig. 3 Force doublets used to express a plastic flow

Hooke's law. Stress fields due to point force doublet can be obtained simply by differentiating that of due to a point force with respect to coordinate variables. Fig. 3 shows three types of force doublets used to express a plastic flow. Magnitudes of force doublets of tension types $T_{x x}, T_{y y}$ and shear type $T_{x y}$ are defined as follows.

$$
T_{x x}=\lim _{\delta \rightarrow 0}\left(\delta \times F_{x}\right), T_{y y}=\lim _{\delta \rightarrow 0}\left(\delta \times F_{y}\right), T_{x y}=\lim _{\delta \rightarrow 0} \delta \times\left(F_{x}+F_{y}\right)
$$

The complex potentials of the force doublet which correspond to the Kelvin solution can be expressed as follows.

$$
\begin{aligned}
& \Omega_{d}(z)=\frac{T_{x x}-T_{y y}}{2 \pi(\kappa+1)} \frac{1}{z-\zeta} \\
& \omega_{d}(z)=-\frac{\kappa-1}{2 \pi(\kappa+1)} \frac{T_{x x}+T_{y y}}{z-\zeta}+\frac{T_{x x}-T_{y y}+2 i T_{x y}}{2 \pi(\kappa+1)} \frac{\bar{\zeta}}{(z-\zeta)^{2}}
\end{aligned}
$$

In the next section, the line weld model and its thermoelastic solution is discussed. Then the procedure for treating a thermoplastic strain is described under the assumption that the material follows Prandtl-Reuss flow rule for an elastic-perfect-plastic body under plain strain condition.

\section{A simplified model for line welding}

Fig.4 shows simplified welding model. A uniform strength of transient line heater of width " $w$ " is applied to a surface of a semi-infinite medium for a short duration of time with a strength chosen so that the total heating energy delivered from the heater resembles to that of expected under actual welding of stainless steel. In a physical sense, the problem is essentially two dimensional which should simplify the analysis, however, an occurrence of residual stress in the out-of-plane $(z)$ direction becomes significant. The resulted thermoelastic field such as temperature rise $\tau(x, y, t)$ and thermoelastic stress components $\sigma_{i j}(x, y, t)$ due to a heating of duration $t$, can be written under the assumption of plane strain $\left(\varepsilon_{z z}=0\right)$ that,

$$
\tau(x, y, t)=\frac{Q}{2 \pi \lambda} \int_{-\frac{w}{2}}^{\frac{w}{2}} E_{1}(S) d \xi
$$




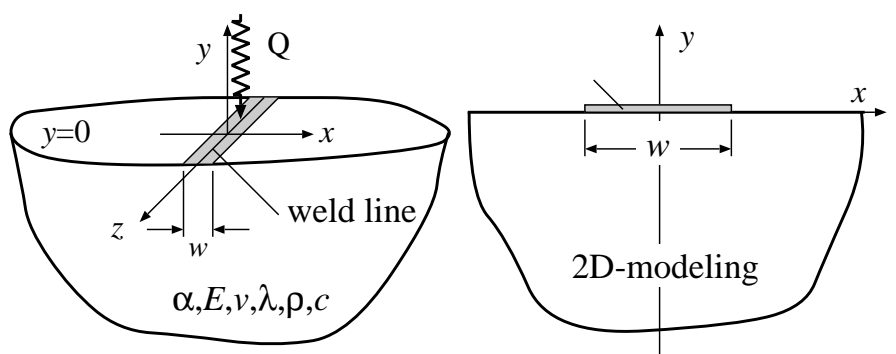

Fig. 4 Schematic illustration of treated problem ( yield stress $\sigma_{Y}=800 \mathrm{MPa}$, heating power $Q=11.2 \mathrm{MW} / \mathrm{m}^{2}$, coefficient of linear expansion $\alpha=1.2 \times 10^{-5} 1 / \mathrm{K}$, Young's modulus $E=200.2 \mathrm{GPa}$, mass density $\rho=7833 \mathrm{~kg} / \mathrm{m}^{3}$, specific heat $c=$ $586 \mathrm{~J} / \mathrm{kgK}$, Poisson's ratio $\nu=0.3$ and thermal conductivity $\lambda=52 \mathrm{~W} / \mathrm{mK}$ )

$$
\begin{aligned}
\frac{\sigma_{x x}(x, y, t)}{\bar{\sigma}} & =\int_{-\frac{w}{2}}^{\frac{w}{2}}\left(2 \frac{y^{2}}{r^{2}}-1\right) \frac{1-e^{-S}}{S} d \xi-\int_{-\frac{w}{2}}^{\frac{w}{2}} E_{1}(S) d \xi \\
& -\frac{2 y}{\pi} \int_{-\infty}^{\infty} \frac{(x-\xi)^{2}}{r^{4}} f(\xi, t) d \xi \\
\frac{\sigma_{y y}(x, y, t)}{\bar{\sigma}} & =\int_{-\frac{w}{2}}^{\frac{w}{2}}\left(1-2 \frac{y^{2}}{r^{2}}\right) \frac{1-e^{-S}}{S} d \xi-\int_{-\frac{w}{2}}^{\frac{w}{2}} E_{1}(S) d \xi \\
& -\frac{2 y^{3}}{\pi} \int_{-\infty}^{\infty} \frac{f(\xi, t)}{r^{4}} d \xi \\
\frac{\sigma_{x y}(x, y, t)}{\bar{\sigma}} & =2 y \int_{-\frac{w}{2}}^{\frac{w}{2}} \frac{x-\xi}{r^{2}} \frac{1-e^{-S}}{S} d \xi-\frac{2 y^{2}}{\pi} \int_{-\infty}^{\infty} \frac{x-\xi}{r^{4}} f(\xi, t) d \xi \\
\frac{\sigma_{z z}(x, y, t)}{2 \bar{\sigma}} & =-\int_{-\frac{w}{2}}^{\frac{w}{2}} E_{1}(S) d \xi-\nu \frac{y}{\pi} \int_{-\infty}^{\infty} \frac{f(\xi, t)}{r^{2}} d \xi
\end{aligned}
$$

where $r^{2}$ is a square of distance between reference and source points $r^{2}=$ $(x-\xi)^{2}+y^{2},(x, y)$ is a coordinate of reference point, $(\xi, 0)$ is a coordinate of source point, $\bar{\sigma}$ is a constant defined by $\bar{\sigma}=\alpha E Q / 4 \pi \lambda(1-\nu)$ in which $\rho$ is a mass density, $c$ is a specific heat, $\lambda$ is a thermal conductivity, $\alpha$ is a coefficient of linear expansion, $E$ is a Young's modulus, $\nu$ is a Poisson's ratio. $S$ is a non-dimensional parameter defined by $S=\rho c r^{2} / 4 \lambda t, E_{1}(x)$ is a integral exponential function defined as

$$
E_{1}(x)=\int_{x}^{\infty} \frac{e^{-u}}{u} d u
$$

and $f(\xi, t)$ is a function defined as

$$
f(\xi, t)=2 \sqrt{\frac{\lambda t}{\rho c}} \times\left[\frac{1}{p}\left(1-e^{-p^{2}}\right)+p E_{1}\left(p^{2}\right)\right]_{p_{1}}^{p_{2}}
$$

where $p_{1}=\sqrt{\frac{\rho c}{\lambda t}} \frac{\xi+w / 2}{2}$ and $p_{2}=\sqrt{\frac{\rho c}{\lambda t}} \frac{\xi-w / 2}{2}$. 

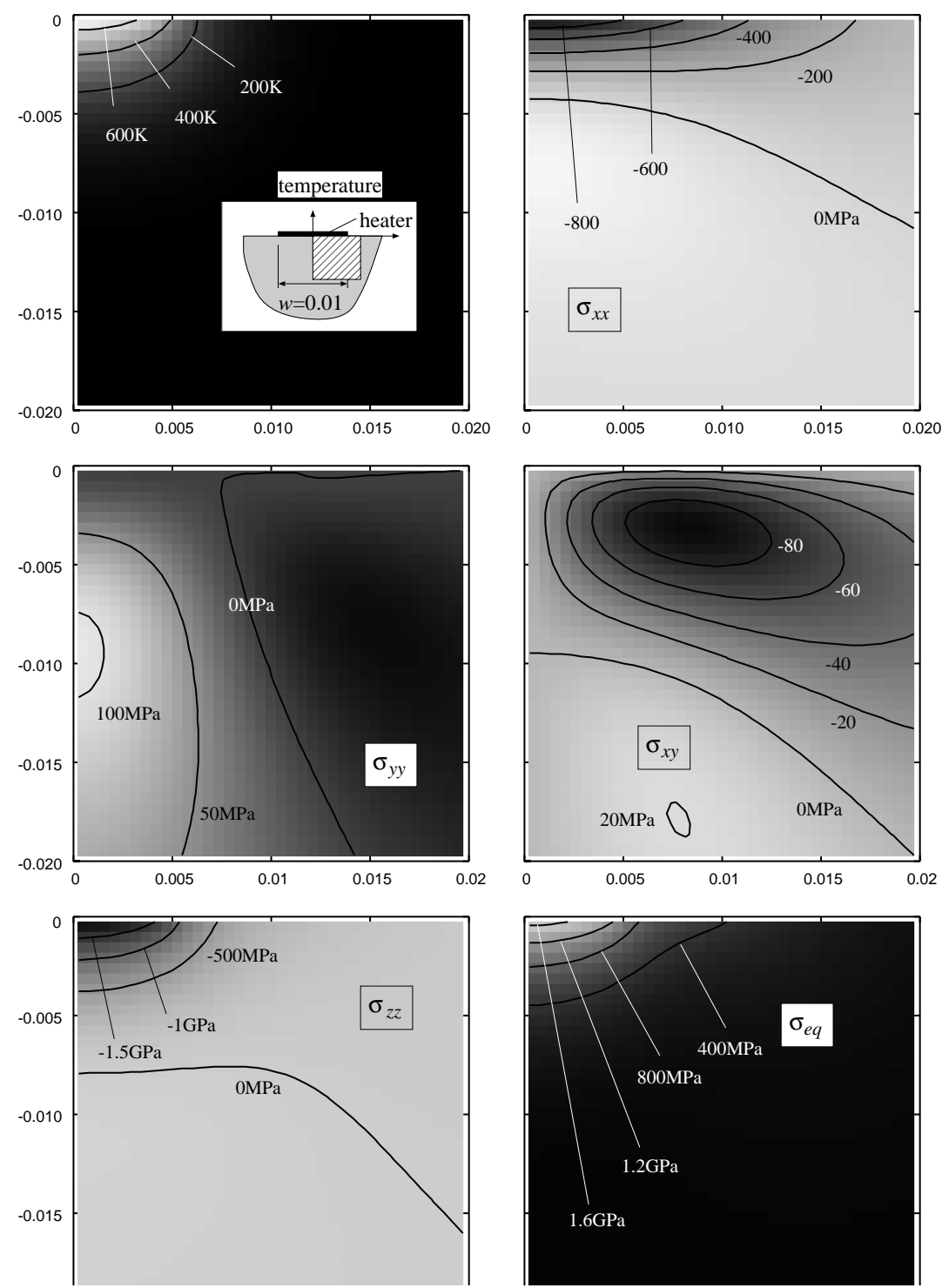

Fig. 5 Thermoelastic fields of welding model at heating time $t=1 \mathrm{~s}\left(\sigma_{\text {eq }}\right.$ is a equivalent stress defined by Eq.(33))

In the present sample, the heating width is set $w=10 \mathrm{~mm}$ and heating duration $t=1 \mathrm{~s}$. In Fig.5, the temperature and the thermoelastic stress distribution at time $t=1 \mathrm{~s}$ are shown as an example, which can be evaluated from Eqs. $(21) \sim(25)$. 


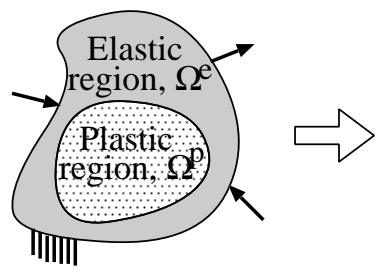

(a) elastic-plastic body

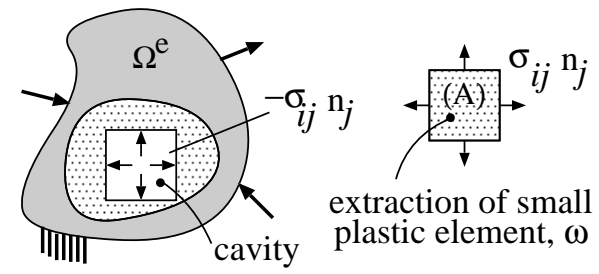

(b) extraction of yielded element without changing stresses

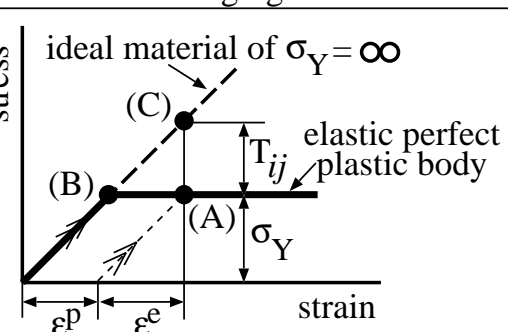

(c) replacement to elastic element

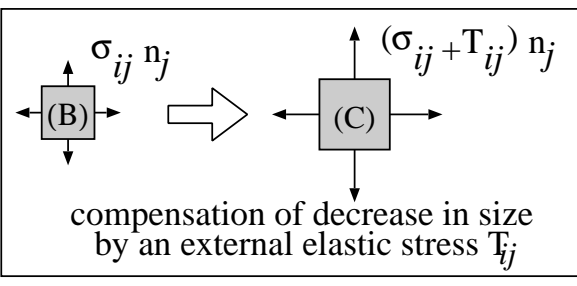

(d) compensation of element shrinkage

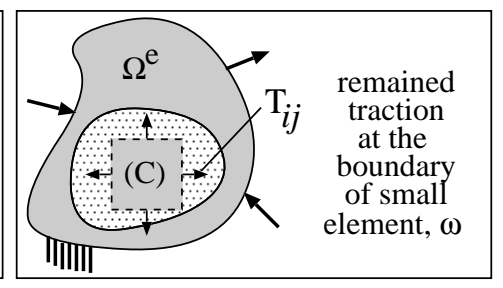

(e) partially replaced body

Fig. 6 Procedure for the replacement of plastic strain $\varepsilon_{i j}^{p}$ by force doublet of magnitude $T_{i j}$

\section{Expression of the plastic strain by force doublets}

The most fundamental concept for the treatment of plastic strain in BFM is to replace the distribution of plastic strain by continuously embedded force doublets [4]. Consider an elastic-plastic body whose elasticity constants are $E$ for Young's modulus and $\nu$ for Poisson's ratio. The plastic part in the region is named $\Omega^{p}$ which is surrounded by an elastic foundation $\Omega^{e}$ as seen in Fig.6(a). Next, consider an infinitesimally small plastic element $\omega$ which has stress components $\sigma_{i j}(P)$ and strain components $\varepsilon_{i j}(P)=\varepsilon_{i j}^{e}(P)+$ $\varepsilon_{i j}^{p}(P)$ where $P\left(x_{1}, x_{2}\right)$ is a reference point in the infinitesimal element $\omega$, $\varepsilon_{i j}^{e}(P)$ and $\varepsilon_{i j}^{p}(P)$ are the elastic and plastic components of strain at point $P$, respectively. $\omega$ can be extracted without affecting the stress field if traction $t_{i}(P)=\sigma_{i j}(P) n_{j}(P)$ is applied to the outer surface of $\omega$, and at the same time, traction $-t_{i}$ is applied to the inner surface of the cavity which is made by the extraction of $\omega$ from $\Omega^{p}$ where $n_{j}(P)$ is a component of unit outward normal. Then the plastic element $\omega$ is transposed to an ideal elastic element $\omega^{e}$ which has the same elastic properties $(E, \nu)$ with external elastic region 
$\Omega^{e}$ but its yield stress $\sigma_{Y}$ is assumed to be infinite so that no yielding takes place. Owing to this transposition (from state (A) to state (B) in Fig.6(c)), stress state is unchanged but the strain state is decreased by the amount of plastic strain $\varepsilon_{i j}^{p}(P)$. Therefore, if $\omega^{e}$ is embedded into the cavity of the region $\Omega^{p}$, some clearances due to shrinkage of the element would be observed. In order to compensate this strain decrease and to embed an ideal element without any gap, an additive stress $T_{i j}(P)$ have to be applied (state $(\mathrm{C})$ in Fig.6(d)) to $\omega^{e}$. This replacement procedure is continued until all the plastic element are transposed to an ideal elastic one. After the completion of such transposition, the stress field at an arbitrary point $P$ can be expressed as follows.

$$
\sigma_{i j}(P)=\sigma_{i j}^{\mathrm{therm}}(P)+\iint_{\Omega} \sigma_{i j}\left(P, Q_{k \ell}\right) T_{k \ell}(Q) d \Omega^{p}(Q)-T_{i j}(P)
$$

where $\sigma_{i j}^{\text {therm }}(P)$ is component of thermoelastic stresses at point $P$ which is shown from Eqs. $(22) \sim(25), Q\left(\xi_{1}, \xi_{2}\right)$ is a source point, $\sigma_{i j}\left(P, Q_{k \ell}\right)=$ $\partial \sigma_{i j}\left(P, Q_{k}\right) / \partial \xi_{\ell} . T_{i j}(Q)$ is a magnitude of force doublet embedded at point $Q$, which compensate the strain decrease during the process of transposition from plastic element $\omega$ to elastic one $\omega^{e}$. Because of the incremental nature of plasticity, not the total stress but an incremental stress is used to evaluated a present stress state. Then Eq.(28) is replaced by an incremental form as

$$
d \sigma_{i j}(P)=d \sigma_{i j}^{\text {therm }}(P)+\iint_{\Omega} \sigma_{i j}\left(P, Q_{k \ell}\right) d T_{k \ell}(Q) d \Omega^{p}(Q)-d T_{i j}(P)
$$

in which $d T_{i j}(P)$ is an increment of the magnitude of force doublet, which is related to the increment of plastic strain at point $P$. The total stress can be calculated by a sum of stress increments such that

$$
\sigma_{i j}(P)=\sum d \sigma_{i j}(P)
$$

When Prandtl-Reuss flow rule is employed, each component of plastic strain increment is assumed to be proportional to a component of deviatoric stress $S_{i j}$ with unknown proportionality constant $d \lambda$. Therefore, the increment of the magnitude of point force doublet can be expressed as

$$
d T_{i j}(Q)=D_{i j k \ell} d \varepsilon_{k \ell}^{p}(Q)=D_{i j k \ell}\left(\sigma_{k \ell}(Q)-\delta_{k \ell} \frac{\sigma_{m m}(Q)}{3}\right) d \lambda(Q)
$$

where $D_{i j k \ell}$ is an elastic modulus tensor and $\delta_{i j}$ is Kronecker delta. It should be noted that the term " $-d T_{i j}(P)$ " in Eq. $(29)$ is indispensable with no relation to the value of $\sigma_{i j}\left(P, Q_{k \ell}\right)$. In fact, stress components due to point force doublet which acts in the $z$ direction $\sigma_{i j}\left(P, Q_{z z}\right)$ results no influence at any point $P$ under plane strain condition. However, even when $\sigma_{i j}\left(P, Q_{k \ell}\right)=0$ for points $(P \neq Q)$, the term $-d T_{i j}(P)$ still yields a non-zero influence at point $P$. In a practical analysis, the proportional constant $d \lambda(Q)$ in Eq.(31) is the unknown parameter to be determined through numerical analysis. Since $d \lambda(Q)$ is not only a function of the position $Q$ but also the function of time $t$, it is required to determine the value of $d \lambda(Q)$ step-wisely, considering the 


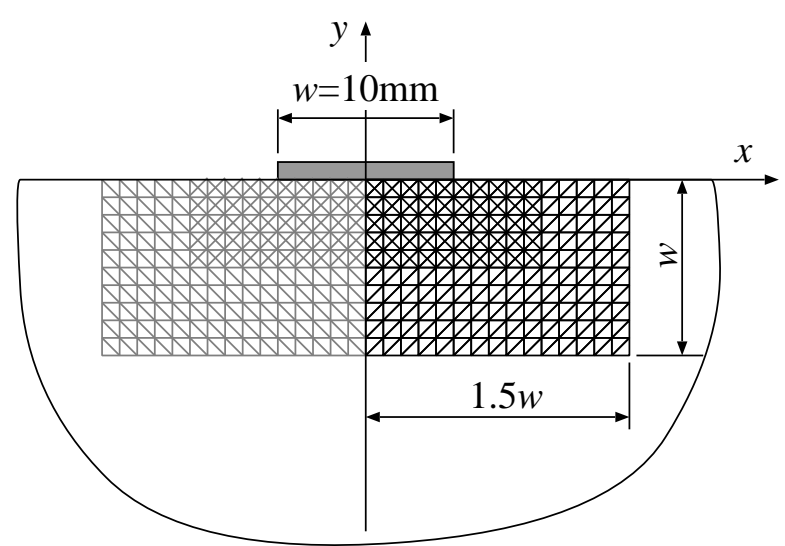

Fig. 7 A regular triangular mesh used to determine unknown parameter considering a symmetry respect to $y$ axis

yield criterion. For example, when Von Mises criterion for elastic-perfectplastic body is supposed, the following equation must hold at an arbitrary point $P$ in the yielded region.

$$
\sigma_{\mathrm{eq}}=\sigma_{Y}
$$

where $\sigma_{Y}$ is a yield stress measured under single axial tensile test and $\sigma_{e q}$ is a equivalent stress defined by

$$
\sigma_{\mathrm{eq}}=\sqrt{\frac{1}{2}} \sqrt{\left(\sigma_{x x}-\sigma_{y y}\right)^{2}+\left(\sigma_{y y}-\sigma_{z z}\right)^{2}+\left(\sigma_{z z}-\sigma_{x x}\right)^{2}+6 \sigma_{x y}^{2}}
$$

\section{Numerical procedure and discussion}

For the numerical estimation of plastic strain, the time domain is divided into $\mathrm{N} t h$ equally division as $t=n \Delta t,(n=1,2, \cdots, N)$ where $\Delta t$ is a time increment. A space domain is also divided into number of triangular areas, which may be generated by the use of an automated Delaunay tessellation scheme in general. For the sake of simplicity, the magnitude of plastic strain (and therefore the magnitude of force doublet) is assumed to be constant over a triangular element at each time step. In the present analysis, however, by considering the symmetry of the problem, a regular set of triangular elements as shown in Fig.7 were employed to evaluate the plastic strain. That is, the equivalent stress at the centroid of each triangular element is used for the determination of $d \lambda(Q)$.

As a result, the total stress component at the reference time $t=n \Delta t, \sigma_{i j}^{n}(P)$ can be evaluated as

$$
\begin{aligned}
\sigma_{i j}^{n}(P) & =\sigma_{i j}^{n-1}(P)+\left.d \sigma_{i j}^{\text {therm }}(P)\right|_{t=n \Delta t} \\
& +\iint_{\Omega} \sigma_{i j}\left(P, Q_{k \ell}\right) d T_{k \ell}^{n}(Q) d \Omega^{p}(Q)-d T_{i j}^{n}(P)
\end{aligned}
$$


where $d T_{i j}^{n}(P)$ is an increment of the magnitude of force doublet at time $t=n \Delta t$. As seen Eq. $(31), d T_{i j}^{n}(P)$ is related to the total stress state at $t=n \Delta t$ but it could be reasonable to evaluate its value from the value of total stress at one time step $\Delta t$ before. That is, $d T_{i j}^{n}(P)$ is approximated such that

$$
d T_{i j}^{n}(P) \approx D_{i j k \ell}\left(\sigma_{k \ell}^{n-1}(P)-\delta_{k \ell} \frac{\sigma_{m m}^{n-1}(P)}{3}\right) d \lambda^{n}(P)
$$

in which $d \lambda^{n}(P)$ is unknown parameter yet not determined. Substitution of Eq.(35) into Eq.(34) leads stress components at arbitrary point $P$ at reference time $t=n \Delta t$, if parameter $d \lambda^{n}(P)$ is given. In order to determine $d \lambda^{n}(P)$, the yield criterion is applied. However, substitution of Eq.(34) into Eq.(32) through Eq.(33) leads nonlinear simultaneous equations for the determination of $d \lambda^{n}(P)$ at each reference point $P$. These nonlinear simultaneous equations should be solved carefully under the constraint that $d \lambda^{n}(P) \geq 0$. When $d \lambda^{n}(P)$ becomes negative, it means the unloading process during plastic deformation so that the value of $d \lambda^{n}(P)$ should be set at 0 . In Figs. 8 and 9 the residual stress distribution along the $y$ and $x$ axes are shown. As seen, the out-of-plane residual stress component $\sigma_{z z}$ exhibits the largest component and the usual plane strain relation $\sigma_{z z}=\nu\left(\sigma_{x x}+\sigma_{y y}\right)$ does not hold.

In the present analysis, the yield stress $\sigma_{Y}$ is assumed to be constant at $800 \mathrm{MPa}$ irrespective to the temperature. Even after the complete cooling, severe residual stresses can be found near the heated zone. For example, the residual stresses at the origin are about $\sigma_{x x}=362 \mathrm{MPa}$ and $\sigma_{z z}=917 \mathrm{MPa}$ as seen in Fig.8. It means that the origin is still in the state of yielding since the equivalent stress $\sigma_{\text {eq }}$ is almost equal to $\sigma_{Y}=800 \mathrm{MPa}$.

\section{Conclusion}

A method for a treatment of limited plastic flow in the body force method was reviewed briefly and a treatment of plastic strain in the direction of out-of-plane based on the principle of the superposition was discussed. In a numerical example, a treated material is assumed an elastic-perfect-plastic body that follows Von Mises yield criterion. It was found that the residual stress in the out-of-plane direction $\sigma_{z z}$ can be estimated independently of the in-plane residual stress components $\sigma_{x x}$ and $\sigma_{y y}$. It was also found that the proposed method provides effective and efficient scheme for treating a problem of limited plasticity.

\section{References}

1. P.M. Blomerus and D.A. Hills, An efficient procedure for modeling limited plastic flow, Proc. Inst. Mech. Eng. C, 212, p.731 (1998)

2. D.H. Chen and H. Nisitani, Extension of Body Force Method to Elastic-Plastic Problems, Transaction of the Japan Society of Mechanical Engineers, Ser. A, 51-462, p.571, 1985 (in Japanese)

3. H.Nisitani and D.H. Chen, Elastic-Plastic Analysis of an Infinite Plate with an Elliptic Hole by Body Force Method, Transaction of the Japan Society of Mechanical Engineers, Ser. A, 51-465, p.1471, 1985 (in Japanese) 


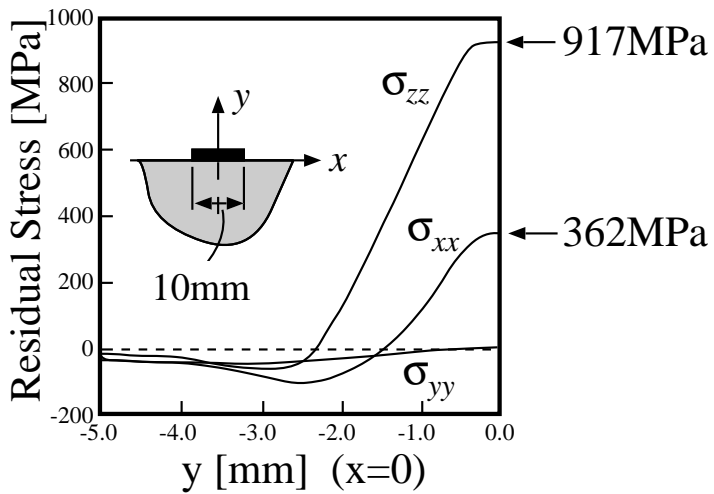

Fig. 8 Residual stress along $y$ axis after cooling

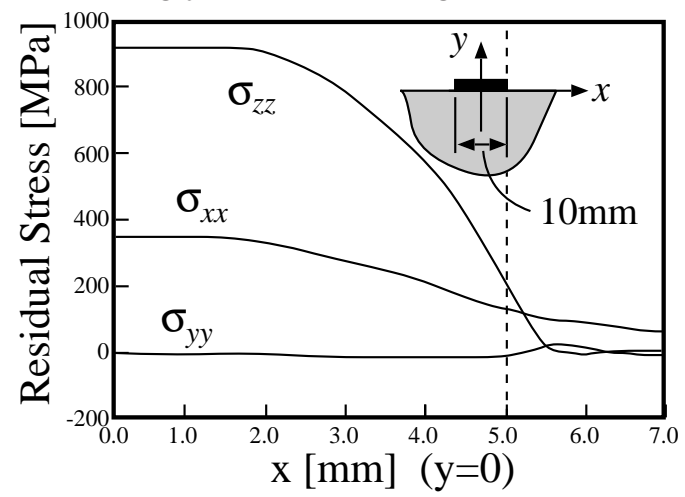

Fig. 9 Residual stress along $x$ axis after cooling

4. D.H. Chen and H.Nisitani, Body Force Method, International Journal of Fracture, Vol. 86, No.1-2, p.161, 1997

5. H.Nisitani, The Two-Dimensional Stress Problem Solved Using an Electric Digital Computer, Bulletin of Japan Society of Mechanical Engineers, Vol.11, No.43, p.14, 1968 